\title{
PRECIOS PSICOLÓGICOS Análisis de la Percepción del Consumidor
}

MBA - SORAYA CASTRO HERRERA(*)

MBA-ALEJANDRO ROSENBERG (**)

\section{INTRODUCCIÓN}

En la mayoría de los comercios minoristas y mayoristas, las decisiones de fijación de precios es el tema más difícil e importante del marketing. Más aún, cuando se suelen introducir ciertas diferencias entre los productos para cada establecimiento en función de las características de los mercados locales (coste de transporte, de operación e implantación, imagen, competencia, etcétera).

La teoría tradicional de la determinación de los precios se apoya en la existencia de clientes bien informados sobre niveles de precios. Sin embargo, en la práctica esta sentencia no necesariamente se cumple, debido a los efectos de diferenciación de productos, la proliferación de marcas de un mismo tipo de producto con precios diferenciados, la existencia de envases de contenidos distintos que dificulta la comparación, los productos que presentan múltiples dimensiones y atributos, lealtad a la marca, los procesos inflacionarios, entre otros.

Los consumidores -según trabajos empíricos-tienen dificultad para valorar la calidad de un producto, por lo que tienden a considerar que los productos de mayor precio suelen ser también los de mayor calidad. Asimismo, ellos eligen el establecimiento en el que realizan sus compras en un ámbito territorial definido, por lo general en las proximidades a su domicilio, con lo que tan solo le resultará relevante la información sobre precios de los establecimientos que forman parte de su conjunto elegible. También se debe considerar los cambios en los hábitos de vida y el menor tiempo disponible para la compra.

Todo lo mencionado contribuye a que exista un menor tiempo disponible para obtener información respecto a los precios. El consumidor por comodidad preferirá comprar todos los productos en un único establecimiento, antes de repartir sus compras entre los establecimientos que ofrecen sus productos a un menor precio.

Estas carencias de información asumen que el consumidor, en la mayoría de los casos; formen sus propios juicios de valor y tomen decisiones cuando no tienen la información perfecta. Y es tarea del marketing presentar la información de precios necesaria para generar nuevos juicios (percepciones) de valor en los compradores, y así, conducirlas a una mayor intención de compra.

El presente estudio está enfocado en la fijación de precios desde el punto de vista de la percepción psicológica de los consumidores ante una asumida carencia de información-detallada líneas arriba-. Nos referi-

(*) Docente de la Facultad de Ciencias Contables de la Universidad Nacional Mayor de San Marcos y Doctoranda de la Universidad Autónoma de Madrid, España.

(**) Doctorando de la Universidad Autónoma de Madrid, España. Nacionalidad chilena. 
remos, especialmente, al tema de los precios psicológicos que han tenido una amplia utilización en el planteamiento de estrategias minoristas. Así también, plantearemos las diversas estrategias y desarrollaremos un caso práctico aplicando la estrategia de cambio en el número de los dígitos.

\section{PRECIO Y PERCEPCIONES DE VALOR DE LOS CONSUMIDORES}

La literatura reciente sobre el comportamiento ha llevado a explicaciones de cómo las personas forman juicios de valor y toman decisiones cuando no tienen información perfecta. Estos nuevos desarrollos permiten entender por qué los consumidores son más sensibles a ciertas modificaciones de los precios (incrementos o reducciones). La conclusión de estos trabajos arroja que los compradores juzgan los precios comparativamente, es decir, el precio de referencia condiciona sus juicios.

\section{Precios de referencia}

Un precio de referencia puede ser un precio externo (precio de un producto sustituto), un precio interno (recuerdo de la última compra realizada), un precio esperado o alguna creencia sobre el precio de un producto en el mismo mercado.

La teoría de adaptación nos dice que no sólo hay un precio de referencia; sino que este cambia. El precio de referencia está aceptado por efectos contextuales tales como frecuencia de cambios previos del precio, expectativas de los compradores acerca de los precios futuros, orden en que la información de precios se presenta a los compradores, la publicidad sobre precios, intensidad de los precios de promoción, etc.

La teoría del contraste -asimilaciónindica que existe una amplitud de captación de precios y que es probable la presencia de un rango de precios alrededor del precio de referencia; en el cual, el precio resultante de un cambio en el precio inicial puede no ser percibido como muy diferente.

Por su parte, el argumento psicológico reconoce que las personas responden de forma diferente a ganancias percibidas y a pérdidas percibidas y sugiere que los compradores son más sensibles a incrementos de precios que a disminuciones de precios.

\section{La percepción}

La percepción es el proceso de categorización de los compradores, los cuales tienden a colocar nuevas experiencias en la clasificación existente de experiencias familiares. Si los compradores se enfrentan a un precio diferente del que pensaban, deberán decidir si la diferencia entre el nuevo y el viejo precio es significativa o no. Si la diferencia es insignificante clasificarán los precios (nuevo y viejo) como similares y no modificarán su patrón de comportamiento. Si la diferencia es significativa, cambiarán su comportamiento de compra y clasificarán al nuevo precio en una nueva categoría de producto-precio.

\section{Efectos de los precios de venta en las percepciones de valor del consumidor}

Numerosas variables de decisión ayudan a comprender cómo los precios influyen en las percepciones de valor y elección del producto. Por ejemplo, el contexto de la decisión de compra y cómo es presentada la oferta que condiciona la evaluación del comprador y la disponibilidad de la información. Al respecto, esta última ción que es fácilmente retenida en la memoria, tanto por su novedad como por el fuerte impacto, influirá en la toma de decisiones. Por otra parte, el orden de presentación de 
los precios establece el juicio de los consumidores sobre los precios altos o bajos en una línea de productos. En la práctica, numerosos vendedores tienden a elevar su precio de lista en espera que una futura negociación de precios con el comprador. El alto precio inicial sirve como "ancla" y el precio negociado, generalmente, es mayor del que debería haber sido sin este precio inicial de referencia. Finalmente, las personas eligen a menudo una alternativa que asocian con algún éxito pasado, o rechazan aquellos asociados a fracasos previos.

\section{Precio y valor del producto}

La figura $\mathrm{N}^{\circ} 1$ ilustra el papel del precio en las percepciones de los compradores en calidad del producto, sacrificio, valor y disponibilidad a pagar. Este modelo sugiere que los compradores no usan el precio sólo como una medida de sacrificio, sino también como un indicador de la calidad del producto o servicio. consumidores toman sus decisiones de compra en un proceso de dos etapas: primero, juzgan el valor de una oferta; $y$ segundo, deciden si hacen la compra; o que pospongan la compra en busca de una mayor información.

Los consumidores evalúan los precios comparando éstos o bien con otros precios posibles de comparación o con precios de referencia que están almacenados en la memoria. Puesto que los precios son evaluados comparativamente, el juicio de aceptabilidad no sólo depende de las expectativas de precio de los consumidores, sino también de la información proporcionada en promociones $\mathrm{o}$ anuncios publicitarios. $\mathrm{La}$ percepción del ahorro transmitido por el precio anunciado lleva a una respuesta positiva del comportamiento.

\section{Interrelación precio - valor percibido}

El precio percibido se descompone en

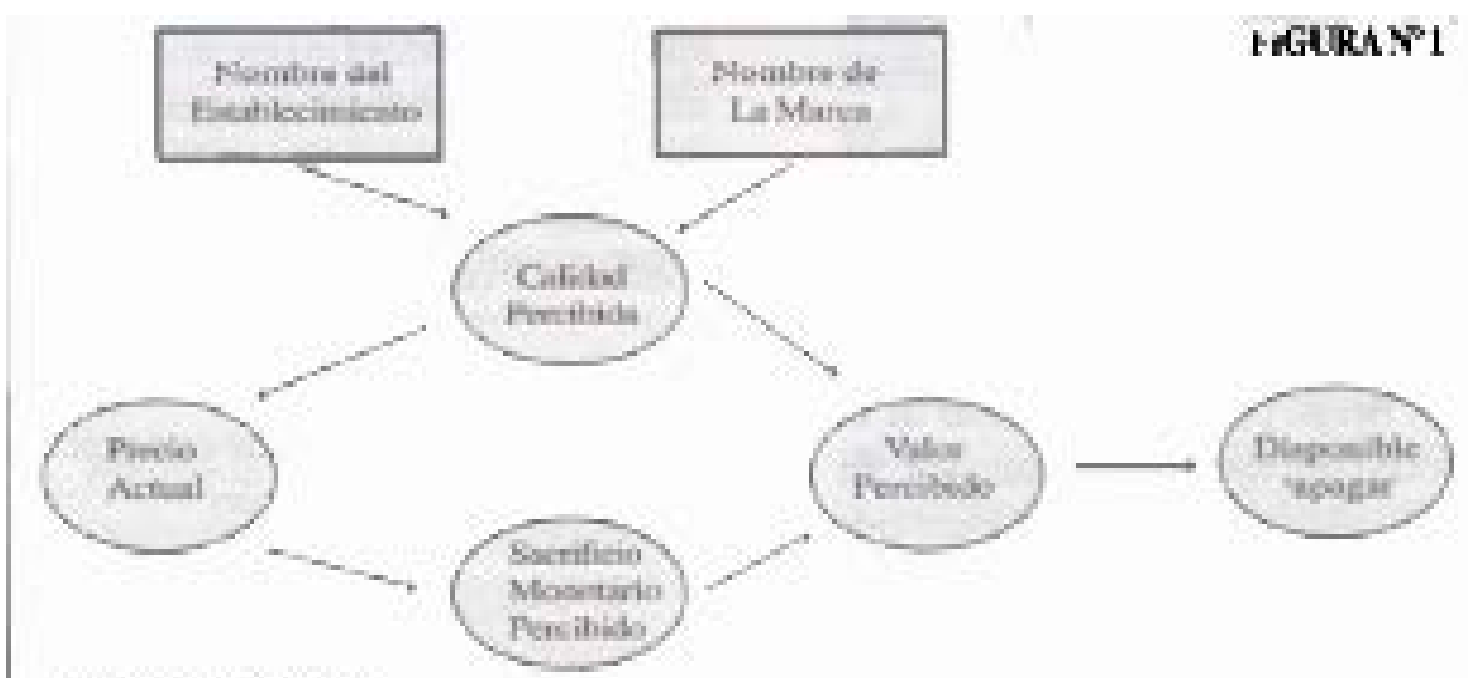

Fuenie: K B Munme

La percepción de los consumidores de un precio deriva de su interpretación sobre las diferencias de precios (reales o implícitas) y de sus interpretaciones de las señales focales y contextuales de la oferta. Los dos tipos de valoraciones contrapuestas. Por un lado, estaría el sacrificio percibido correlacionado positivamente al precio (a mayor precio mayor sacrificio) y por el otro, la calidad o beneficio percibido que también 
está relacionado directamente con el precio. El conjunto de estas dos variables da lugar al concepto de valor percibido; y la magnitud vendría a estar determinada por los pesos relativos que adquieran estas dos variables. El valor percibido se relaciona positivamente con la intención de compra o disponibilidad para pagar; y ésta a su vez, con el acto de compra.

\section{PRECIOS PSICOLÓGICOS Y SU APLICACION EN MARKETING}

El estudio de la percepción está ligado al análisis del comportamiento de los consumidores y sus aplicaciones en marketing, está asociada a nuestra comprensión del funcionamiento de la percepción. La percepción influye en cualquier decisión de consumo debido a dos razones: los problemas del consumidor son situaciones percibidas, y la decisión, que persiga solucionar un problema, deriva de un proceso de búsqueda de información que es específicamente un proceso de percepción.

En marketing, los aspectos necesarios para trabajar eficazmente partiendo de la comprensión perceptiva es amplia y afecta a las cuatro variables tradicionales: los aspectos relevantes relacionados a la marca son la transmisión de imágenes, el desarrollo de logotipo, etc. En relación al envase, su carácter de vendedor silencioso o la concepción del riesgo percibido derivado de la inseguridad perceptiva del consumidor. En lo referente a la distribución se introduce toda la problemática del diseño de la estrategia minorista con el objetivo de trasmitir una determinada imagen como principal argumento de relación y posicionamiento hacia un determinado segmento de consumidores potenciales; y finalmente, en el área del precio, el conjunto de aplicaciones relacionadas a los precios psicológicos, que es el tema que desarrollaremos en esta sección.

\section{Precios psicológicos}

Según la teoría económica, los precics son los indicadores de costo de un bien $c$ servicio; sin embargo, evidencia empírica análisis psicológico arroja que el precı: transciende las fronteras de lo económico e incluye un elemento psicológico representado por la percepción, con lo que su análisis se torna más complejo. La piedra angular de las estrategias de precios la constituye la forma en que los consumidores perciben loprecios y desarrollan percepciones de valor

a). El precio habitual.- Es el método de fijación de precios donde todos los precios alternativos son excluidos, excepto un único precio. Con precios habituales, los vendedores se adaptan a cambios en los costes y condiciones de mercado; asimismo. asumen que el consumidor considera pagar solo un precio.

La práctica de este sistema consiste en situar el precio de un producto en una cifra exacta, única y duradera; mejor aún si ésta es una partición de la unidad monetaria. Por ejemplo, en Estados Unidos, una goma de mascar normal habitualmente tiene un precio de 15 centavos de dólar. En Perú, un caramelo -cualquiera que sea- tendrá un precio de 20 céntimos.

La literatura reciente indicaría que hoy en día es poco probable que esta práctica tenga mucha aceptación o uso.

b). Precio de línea.- Controlan el precio de un completo inventario de un artículo particular. La mercancía es ofrecida a una cantidad de precios específica y limitada (básicamente tres precios distintos). Los precios pueden mantenerse constantes en un periodo de tiempo y los cambios en las condiciones de mercados se adaptan ajustando la calidad de la mercancía en cada línea. Por ejemplo, una empresa vende una 
línea de helados de chocolate y nata de 100 , 130 y 150 ptas. Los consumidores asumen que el rango 100 y 150 ptas. es equivalente. Por lo tanto, la gama de precio dentro de este rango no debería incrementar la cantidad total vendida.

c).Precio impar,- Son precios con terminación impar o precios por debajo de un número redondo $(99,98,97$.). Estudios al respecto ${ }^{1}$ concluyen que los precios por debajo de un figura redonda $(00,000$, etc.) tienen mayor aceptación, siendo los precios terminados en nueve los de mayor popularidad, seguidos de los precios terminados en cinco. Contrariamente, otras investigaciones ${ }^{2}$ asumen que los precios impares son más difíciles de recordar porque lo que su incidencia positiva en la demanda es poco probable.

Sin embargo, en la práctica, los consumidores aceptan mejor los precios terminados en "9", " 5 " o " 0 " que las otras terminaciones. La explicación en este caso, está sustentada en que los consumidores al leer los precios sólo retienen los primeros dígitos de dicha cifra en su memoria. Por lo que la diferencia percibida entre un precio de terminación nueve y su inmediato superior (cifra redonda) tiende a ser más amplia y exagerada en comparación con la diferencia entre precios situados en una misma escala.

499 vs. 500 comparado con 493 vs. 494

Aunque la diferencia es la misma, la percepción entre las diferencias de cada par de precios es diferente. Esta estrategia de precios es una de las de mayor aceptación (Véase Anexo $\mathrm{N}^{\circ} 1$ ). Independientemente del sector, las terminaciones en número impar "9", " 5 " u "0" son utilizadas por excelencia. d). Cambio en el número de los dígitos.- Una reducción o aumento en el precio que implica un cambio en el número de dígitos del precio será percibida como mayor en comparación con una disminución o elevación de precios en el que el cambio de dígitos no existe. Por ejemplo:

950 a 935 comparado con 1010 a 995

915 a 930 comparado con 990 a 1005

La reducción (aumento) en todos los casos es de 15 pesetas en el precio. En el primer caso se ha mantenido el número de dígitos de tres, en cambio, en el segundo de dígitos ha bajado de cuatro a tres. Empíricamente se ha demostrado que aunque la variación-reducción o aumento-del precio es la misma, el efecto en la baja de precios, cuando se incluyen cambios en los dígitos, es mayor dado que la distancia psicológica en los casos de la derecha es substancialmente notable.

\section{e).Estrategia de utilización de} precios de referencia internos.- Tal como mencionamos anteriormente, los precios de referencia internos son precios que el consumidor forma en su mente respecto de cada producto, almacenándolo en su memoria. Es el precio que se considera justo a cada producto o servicio. Consideremos en este acápite el siguiente ejemplo ${ }^{3}$

Se puede ver que en la primera muestra, la mayor parte de los consumidores eligió el microondas Emerson (57\%) por su menor precio. Sin embargo, en la segunda muestra, al agregarse un producto nuevo que es el microondas Panasonic - II, el cual tiene un precio mayor a los otros dos microondas, la mayor parte de los consumidores escogió

1. GABOR y GRANGER Price Sensitivity of the Consumer, 1964.

2. SCHINDLER y WIMAN. Consumer Recall of Odd and Even Prices, 1983.

3. Caso práctico tomado de: Javier Alonso Rivas. Comportamiento del Consumidor. Deccisiones y estrategia de marketing. Y de: Javier Oubiña Barbolla. La Percepción de Precios de los Consumidores. En: Revista Distribución y Consumo, 1997. 
INFLUENCIA DE LOS PRECIOS OBSERVADOS EN LA CATEGORÍA DE PRODUCTOS SOBRE EL PRECIO DE REFERENCIA INTERNO

\begin{tabular}{|l|c|c|}
\hline \multicolumn{1}{|c|}{ TIPOSDEMICROONDAS } & PRECIOS & PORCENTAJES \\
\hline MUESTRA N ${ }^{\circ}$ I & & \\
\hline Microondas Panasonic - I & 179,99 & $43 \%$ \\
Microondas Emerson & 109,99 & $57 \%$ \\
\hline MUESTRA N' 2 & & $13 \%$ \\
\hline Microondas Panasonic - II & 199,99 & $60 \%$ \\
Microondas Panasonic - I & 179,99 & $27 \%$ \\
Microondas Emerson & 109,99 & \\
\hline
\end{tabular}

Fuente: Simonson y Tversky

Tomado de: Alonso Rivas, Javier

el microondas Panasonic - I en versión intermedia que en la primera muestra fuera rechazado por su precio alto.

La posible explicación de este fenómeno es que al ampliarse el rango de referencia interno, el microondas Panasonic - I que en la primera muestra era "caro", ahora resulta más asequible, dado que se presenta un producto de mayor precio y por lo tanto, un límite de referencia superior al anterior. Los consumidores siempre tienden a optar por los precios intermedios (la media). Ni muy caro ni muy barato.

\section{f). Estrategia aplicando los precios} de referencia externos.- Cabe recordar que los precios de referencia externos son los precios que recibe el consumidor a través de distintos canales de información externos y que le ayudan a efectuar comparaciones.

Tomando como referencia el caso anterior, se toma el microondas Emerson: "antes a I09,99 ahora a 105, 99". En este ejemplo, el primer precio aparece como de referencia y así puede conseguirse que el consumidor eleve su precio de referencia interno y perciba el mismo precio ofertado como más atractivo.
g).Estrategia de precios máximos y mínimos.- Normalmente, en la mente de los compradores existe una banda de precios (límites superior e inferior) aceptables para el nivel de calidad del bien o servicio al que enfrenta su decisión de compra. Los precios que no pertenecen a dicha banda son considerados insatisfactorios y rechazados.

Una empresa que por su estructura interna pueda reducir sus precios debe saber hasta qué nivel mínimo hacerlo, si rebasa el umbral inferior probablemente el precio sea considerado como no aceptable por los consumidores que dejaran de comprar la marca. Asimismo, la estrategia de precios altos para transmitir una imagen de marca de calidad tiene su límite en el umbral superior.

h). Precio conjunto.- También denominado precio de paquete. Es una estrategia de precios que consiste en ofrecer dos o más bienes o servicios a un único precio, que lógicamente será menor que la suma de los precios unitarios. Los ejemplos típicos de esta estrategia son: los paquetes turísticos, las ventas promocionales de videos o música clásica, introducción de nuevos productos, etc. El problema con esta estrategia es que hay diferentes segmentos de consumidores que valoran de manera distinta las combina- 
ciones de productos o servicios, en otras palabras sus percepciones de valor son diferentes. Cada segmento está dispuesto a pagar cantidades máximas por cada producto incluido en el paquete.

\section{i). Respuestas asimétricas al precio.-} Los compradores perciben de manera diferente las ganancias y pérdidas de igual magnitud. Casi siempre respondemos desfavorablemente ante un aumento de precio que favorablemente ante una disminución igual del precio.

Un ejemplo muy regular al respecto es: dos tiendas que venden el mismo producto. En la primera tienda, la información relacionada a una guitarra es la siguiente:

$$
\begin{array}{|lr}
\hline \text { Precio de la guitarra } & 300.000 \text { ptas } \\
\text { Descuento por efectivo } & 30.000 \text { ptas. }
\end{array}
$$

\section{Y en la otra tienda:}

\begin{tabular}{|ll|}
\hline Precio de la guitarra & 270.000 ptas. \\
Pago Financiado & 300.000 ptas.
\end{tabular}

Las condiciones reales por ambos establecimientos son las mismas, pero la información diferente. Ante ambos supuestos, los consumidores optarán por la primera oferta dado que ven una disminución en el precio y se mostrarán desfavorables con la segunda oferta dado que perciben un aumento en el precio.

Asimismo, los consumidores pueden percibir que en la primera oferta se promociona un producto de mejor calidad con descuento, dado que relativamente tiene un precio mayor frente a la segunda opción.

j).Relación precio - calidad.- Los consumidores tienden a utilizar el precio como indicador de calidad. Especialmente, cuando se carece de la información necesaria para una adecuada decisión. Este plantea- miento se encuentra presente en aquellas situaciones que se relacionan con categorías de productos en las cuales existe una mayor variabilidad de precios entre las diferentes marcas y en mucha menor medida cuando las marcas apenas se diferencian por razón de precio.

k). Cambio monedas y billetes.- Está empíricamente demostrado que existe una alta relación entre el valor nominal de un billete y los precios psicológicos. En España, existe el billete de 1.000 pesetas, seguido por uno de 2.000 pesetas. Si existiera un billete de 1.200 pesetas, a lo mejor las empresas podrían con mayor facilidad aumentar sus precios a \$ 1.199 y seguramente la elasticidad de demanda no sería tan sensible.

\section{CASO PRÁCTICO}

Este caso fue tomado de la experiencia de uno de los autores en una empresa multinacional, la cual es líder en Chile en el sector industrial de helados.

En Chile, al igual que en el resto del mundo, compiten fuertemente en el sector de helados industriales, Induslever y Nestlé. Induslever bajo la marca Bresler, y Nestlé bajo la marca Savory, en Chile.

\begin{tabular}{|c|c|}
\hline MARCA & PART. \% DEL MERCADO \\
\hline Savoir & 80 \\
\hline Bresler & 15 \\
\hline Otros & 5 \\
\hline
\end{tabular}

Hasta el año 1988, la proporción de ventas de helados Savory, según los Canales de Distribución se muestra en la siguiente tabla:

\begin{tabular}{|c|c|c|}
\hline Ruta $^{4}$ & Supermercados & Heladerías \\
\hline $80 \%$ & $18 \%$ & $2 \%$ \\
\hline
\end{tabular}

4 Ruta - Pequeños Almacenes (Minimarkets). 
En ese año se vendían aproximadamente 5 millones de litros de helado, con un consumo per-cápita de 2-2,5 lt/persona.

Luego, comenzó en Chile un fuerte crecimiento del canal de distribución "mall" o centros comerciales climatizados. Esto abrió una oportunidad estratégica muy importante, pues desarrolló las heladerías en un ambiente en el cual se podía desestacionalizar el consumo de helados, ya que dentro del centro comercial existía una temperatura constante durante todo el año.

Esto hizo cambiar dos cosas fundamentales, una para la industria del helado, y otra para (Nestlé-Savory) en particular.

Para la industria en general, supuso un aumento espectacular en el consumo de helados per-cápita en Chile, pasando en 1988 de aprox. 2,5 lt/persona a 1998 a 7 lt/ persona. Esto por el gran nivel de comercio que registran los centros comerciales en Chile (ceteris paribus, otras variables importantes, como lo son el aumento del ingreso per-cápita, cambios en patrones de consumo, etc.).

Todo esto también trajo como consecuencia un cambio interno en la importancia relativa de los canales de distribución para Savoy, los cuales se aprecian en la siguiente tabla:

\begin{tabular}{|c|c|c|}
\hline Ruta & Supermercados & Heladerías \\
\hline $74 \%$ & $16 \%$ & $10 \%$ \\
\hline
\end{tabular}

En el año 1998, las ventas de Savory superaban los 100 millones de litros al año, o sea, en 10 años se dobló el consumo de helados Savory en Chile. Los más importantes fueron la tasa de crecimiento de la población en esos diez años de un $2 \%$ anual, lo que nos da un $22 \%$ en ese periodo. El ingreso per-cápita aumentó de US\$5.000 a US $\$ 6.500$, un cambio de un $30 \%$. $^{5}$

\section{Estrategia del producto}

Históricamente el concepto heladería vendía los productos básicos de Savory, lo que son helados de paletas y barquillos.

Desde el año 1993, se introdujeron Copones $^{6}$ de helado para "llevar", aprovechando las sinergías con otras divisiones de Nestlé.

Primero se incorporó la Copa Savory, luego la copa Profiterol, posteriormente la copa Brownie; hoy reemplazada por la copa Tritón, marca de galletas de Nestlé, número uno en la venta de confites; $y$ en general, utilizando constantemente las marcas de Nestlé confites que están muy bien posicionadas en la mente del consumidor.

Hoy día el mix de venta entre paletas, barquillos y copas, y sus relaciones de precio y margen la podemos apreciar en la siguiente tabla.

\section{Estrategia de precios}

El trabajador promedio en Chile cuenta con un presupuesto aproximado de 1.000 pesos $^{7}$ diarios para comer, se intentó hacer algo bastante arriesgado: el de incorporar en la mente del consumidor a través de promoción, publicidad en medios masivos y en el 


\begin{tabular}{|l|l|l|l|l|l|}
\hline & & & & & \\
& Precio c/iva\$ & Precio s/iva\$ & \% Costo & \% Margen & \% de la Venta \\
\hline & & & & & \\
Barquillos & 550 & 466,1 & $34,3 \%$ & $65,7 \%$ & $35,9 \%$ \\
Paletas & 350 (prom) & 296,6 & $54,4 \%$ & $45,6 \%$ & $15,5 \%$ \\
Copas & 999 & 846,6 & $41,0 \%$ & $59,0 \%$ & $45,6 \%$ \\
& & & & & \\
\hline
\end{tabular}

punto de venta que la copa de helado no sólo se podía considerar como un postre, sino que podría ser una comida completa, por sus características nutricionales, tamaño, y prestigio.

El precio elegido para vender, dadas algunas investigaciones de mercado, fue de $\$ 990$, para luego llegar a \$999.

Como referencia podemos mencionar que Mc Donalds tiene una promoción a $\$ 999$, que incluye hamburguesa, patatas fritas y refresco. Al respecto podemos comentar que estudios realizados por Nestlé, comparando aspectos de la calidad percibida entre ambas marcas, evaluadas como muy buenas y valor percibido básicamente dado por la relación calidad percibida y sacrificio percibido, daban una intención de compra, favorable a Mc Donalds, pero seguido de cerca por Savory - Nestlé.

Por otro lado, el precio de la copa de helado obedece a una estrategia de cambios en los números de dígitos. Es decir, el umbral o "techo" del precio quedó claramente manifiesto que es el de $\$ 999$, ya que la elasticidad precio de la demanda, por encima de este umbral, es "elástica", o sea existe una elasticidad precio mayor que la unidad. Esto fue probado al querer en una oportunidad, utilizando un mercado de prueba del barrio "alto" de Santiago, aumentar los precios de las copas de \$999 a \$ 1.099 o sea en un $10 \%$, pero la cantidad de copas vendidas increíblemente cayeron en un $15 \%$, por lo que los ingresos totales cayeron; no en exactamente la diferencia de un $5 \%$, ya que gente que consumía copas comenzó a consumir barquillos y paletas. Esto trajo consigo una caída en las ventas menor al $5 \%$, por un efecto sustitución.

Hay un aspecto clave, el coste para Mc Donalds de una comida completa es de $\$ 500$, mientras que el de una Copa Savory es de $\$ 360$, o sea a igualdad de precios de venta el margen de contribución directo en Savory era de un $28 \%$ mayor al de Mc Donalds.

El competidor directo de Savory, Bresler de Induslever, no se ha mencionado ya que Savory ha colocado muchas barreras de entrada a su competidor en los centros comerciales. En general, Savory estaba sola en la venta de helados, dados acuerdos con las dos cadenas de "malls" más importantes de Chile; las cuales son Parque Arauco S.A y Mall Plaza S.A.

Cabe destacar que la gran ventaja competitiva de Nestlé en las heladerías respecto a Bresler, es que tiene sinergías con su línea de confites, por lo que se puede colocar una marca recordada en la memoria de toda la gente, cosa que no puede hacer Bresler. 
1.- La primera conclusión relevante es que al hablar de precios psicológicos, estamos frente a una realidad diaria que viven los directivos de grandes, medianas y pequeñas empresas.

2.- Este proceso está influenciado por la competencia en cada sector industrial, pero al final los que definen los umbrales o techos para estos precios son los consumidores."

3.- Hay que tener mucho cuidado al elaborar estrategias de precios, en el sentido de obviar este "fenómeno" de los precios psicológicos, ya que para aquellas empresas que no lo consideran desde un primer momento, pueden sufrir importantes inconvenientes con respecto a los consumidores y por ende, a su competencia.

4.- El proceso de referencia del precio psicológico está ligado al consumidor, empresas (competencia), pero según nuestra experiencia, también a la oferta en términos de billetes y monedas que exista en la economía. O sea en el ejemplo que veíamos anteriormente, el billete que existe en Chile es de $\$ 1.000$, seguido por uno de $\$ 2.000$. Si existiera por ejemplo un billete de $\$ 1.200$, a lo mejor las empresas pertenecientes al sector industrial del ejemplo podrían con mayor facilidad aumentar sus precios a $\$ 1.199$ y seguramente la elasticidad de demanda no será tan sensible, como lo es hoy día en Chile.

\section{ALONSORIVAS, Javier}

1997. "Comportamiento del consumidor. Decisiones y estrategia de marketing". Editorial ESIC, Madrid.

CEBOLLADA PASCUAL, Javier 1997. "La gestión de los precios y promociones en la empresa minorista".

En: Distribución y Consumo. Abril - mayo.

CRUZROCHE, Ignacio

1997. "La venta a pérdida en las relaciones Fabricante - Distribuidor". En: Distribución y Consumo. Abril - mayo.

KENK B., Monroe

1992. "Política de precios. Para hacer más rentables las decisiones". Mc Graw Hill.

OUBIÑA BARBOLLA, Javier 1997. "La percepción de precios de los consumidores. Implicaciones para la fijación de precios minoristas". En: Distribución y Consumo. Abril - mayo.

INSTITUTO NACIONAL DE ESTADÍSTICA E INFORMÁTICA - INE. Boletín Mensual.

Diciembre, Chile.

NESTLE

1999. Estudio de mercado de helados. Chile,junio. 